\title{
Journalof the American Chemical Society
}

\section{Article}

\section{Synthesis and Stability of Monolayer-Protected Au38 Clusters}

Outi Toikkanen, Virginia Ruiz, Gunilla Ro\#nnholm, Nisse

Kalkkinen, Peter Liljeroth, and Bernadette M. Quinn

J. Am. Chem. Soc., 2008, 130 (33), 11049-11055 • DOI: 10.1021/ja802317t • Publication Date (Web): 25 July 2008

Downloaded from http://pubs.acs.org on January 14, 2009

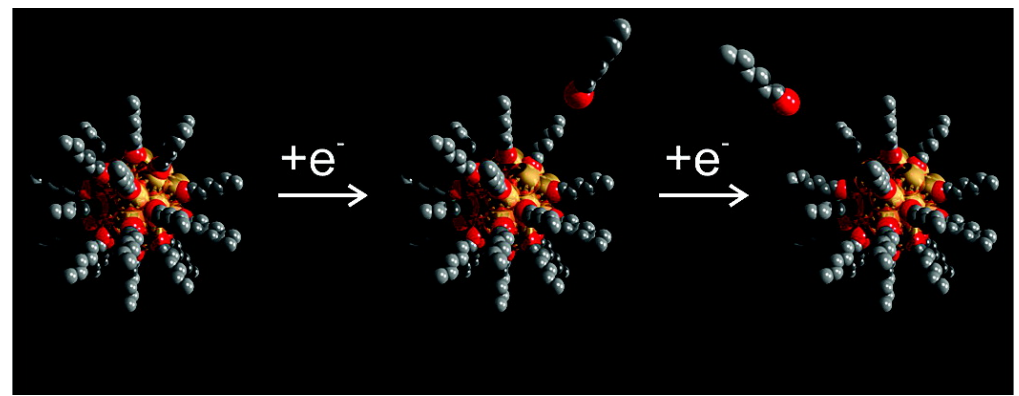

\section{More About This Article}

Additional resources and features associated with this article are available within the HTML version:

- Supporting Information

- Access to high resolution figures

- $\quad$ Links to articles and content related to this article

- Copyright permission to reproduce figures and/or text from this article

\section{View the Full Text HTML}




\title{
Synthesis and Stability of Monolayer-Protected Au38 Clusters
}

\author{
Outi Toikkanen, ${ }^{\dagger}$ Virginia Ruiz, ${ }^{\ddagger}$ Gunilla Rönnholm,${ }^{\S}$ Nisse Kalkkinen, ${ }^{\S}$ \\ Peter Liljeroth," and Bernadette M. Quinn*, \\ Department of Chemistry, Helsinki University of Technology, P.O. Box 6100, FIN-02150 HUT, \\ Finland, Department of Engineering Physics, Helsinki University of Technology, P.O. Box 5100, \\ FIN-02150 HUT, Finland, Institute of Biotechnology, University of Helsinki, P.O. Box 65, \\ FIN-00014, Finland, and Condensed Matter and Interfaces, Debye Institute for Nanomaterials \\ Science, University of Utrecht, P.O. Box 80000, 3508 TA Utrecht, The Netherlands
}

Received March 30, 2008; E-mail: bquinn@ @iki.fi

\begin{abstract}
A synthesis strategy to obtain monodisperse hexanethiolate-protected Au38 clusters based on their resistance to etching upon exposure to a hyperexcess of thiol is reported. The reduction time in the standard Brust-Schiffrin two-phase synthesis was optimized such that Au38 were the only clusters that were fully passivated by the thiol monolayer which leaves larger particles vulnerable to etching by excess thiol. The isolated Au38 was characterized by mass spectrometry, thermogravimetric analysis, optical spectroscopy, and electrochemical techniques giving $\mathrm{Au}_{38}\left(\mathrm{SC}_{6}\right)_{22}$ as the molecular formula for the cluster. These ultrasmall Au clusters behave analogously to molecules with a wide energy gap between occupied (HOMO) and unoccupied levels (LUMO) and undergo single-electron charging at room temperature in electrochemical experiments. Electrochemistry provides an elegant means to study the electronic structure and the chemical stability of the clusters at different charge states. We used cyclic voltammetry and scanning electrochemical microscopy to unequivocally demonstrate that Au38 can be reversibly oxidized to charge states $z=+1$ or +2 ; however, reduction to $z=-1$ leads to desorption of the protecting thiolate monolayer. Although this reductive desorption of thiol from the cluster surface is superficially analogous to electrochemical desorption of planar self-assembled monolayers (SAMs) from macroscopic electrodes, the molecular details of the process are likely to be complicated based on the current view that the thiolate monolayer in clusters is in fact composed of polymeric $\mathrm{Au}-\mathrm{S}$ complexes.
\end{abstract}

\section{Introduction}

Gold nanoparticles coated by an alkanethiol monolayer, socalled monolayer-protected clusters (MPCs), are the focus of intense study due to their interesting optical and electronic properties. ${ }^{1}$ The facile synthesis method developed by Brust et al. in $1994,{ }^{2}$ together with the intrinsic stability of MPCs, started a new field of research. ${ }^{1,3}$ Understanding of the synthesis and properties of MPCs has progressed to the formulaic $\mathrm{Au}_{n}(\mathrm{SR})_{m}$ description, and clusters of a given $n / m$ ratio can be routinely isolated. ${ }^{4}$ These MPCs can be treated as regular chemical reagents with physicochemical properties that can be tuned simply by changing the core size and the nature of the protecting thiol. ${ }^{1}$ MPCs have been proposed for a plethora of applications ranging from sensing to building blocks for the bottom-up assembly of nanoscale devices. $1,3,5$

Due to a combination of nanometer core size and the subattofarad capacitance associated with the protecting mono-

† Department of Chemistry, Helsinki University of Technology.

Department of Engineering Physics, Helsinki University of Technology.

§ University of Helsinki.

"University of Utrecht.

(1) Daniel, M.-C.; Astruc, D. Chem. Rev. 2004, 104, 293.

(2) Brust, M.; Walker, M.; Bethell, D.; Schiffrin, D. J.; Whyman, R. J. Chem. Soc., Chem. Commun. 1994, 801.

(3) (a) Love, J. C.; Estroff, L. A.; Kriebel, J. K.; Nuzzo, R. G.; Whitesides, G. M. Chem. Rev. 2005, 105, 1103. (b) Templeton, A. C.; Wuelfing, W. P.; Murray, R. W. Acc. Chem. Res. 2000, 33, 27. layer, MPCs can demonstrate discrete charging in electrochemical experiments at room temperature. ${ }^{1,3 b, 6}$ Dispersed MPCs have been considered as both diffusing nanoelectrodes and as conventional redox molecules. ${ }^{1}$ Such experiments provide an elegant means of probing the electronic properties of the MPCs as a function of the core size. ${ }^{1,6}$ For MPCs with core radii greater than $1 \mathrm{~nm}$ such as the ubiquitous Au147, charging is controlled by electrostatics, ${ }^{7}$ whereas for clusters with smaller radii such as Au25, MPCs behave analogously to molecules with an energy gap opening between the occupied (HOMO) and unoccupied

(4) (a) Heaven, M. W.; Dass, A.; White, P. S.; Holt, K. M.; Murray, R. W. J. Am. Chem. Soc. 2008, 130, 3754. (b) Tsunoyama, H.; Nickut, P.; Negishi, Y.; Al-Shamery, K.; Matsumoto, Y.; Tsukuda, T. J. Phys. Chem. C 2007, 111, 4153. (c) Shichibu, Y.; Negishi, Y.; Tsunoyama, H.; Kanehara, M.; Teranishi, T.; Tsukuda, T. Small 2007, 3, 835. (d) Negishi, Y.; Chaki, N. K.; Shichibu, Y.; Whetten, R. L.; Tsukuda, T. J. Am. Chem. Soc. 2007, 129, 11322. (e) Jadzinsky, P. D.; Calero, G.; Ackerson, C. J.; Bushnell, D. A.; Kornberg, R. D. Science 2007, 318 , 430. (f) Tsunoyama, H.; Negishi, Y.; Tsukuda, T. J. Am. Chem. Soc. 2006, 128, 6036. (g) Negishi, Y.; Takasugi, Y.; Sato, S.; Yao, H.; Kimura, K.; Tsukuda, T. J. Phys. Chem. B 2006, 110, 12218. (h) Price, R. C.; Whetten, R. L. J. Am. Chem. Soc. 2005, 127, 13750. (i) Negishi, Y.; Nobusada, K.; Tsukuda, T. J. Am. Chem. Soc. 2005, 127, 5261. (j) Donkers, R. L.; Lee, D.; Murray, R. W. Langmuir 2004, 20, 1945. (k) Schaaff, T. G.; Shafigullin, M. N.; Khoury, J. T.; Vezmar, I.; Whetten, R. L.; Cullen, W. G.; First, P. N.; Gutierrez-Wing, C.; Ascensio, J.; Jose-Yacaman, M. J. J. Phys. Chem. B 1997, 101, 7885. (1) Walter, M.; Akola, J.; Lopez-Acevedo, O.; Jadzinsky, P. D.; Calero, G.; Ackerson, C. J.; Whetten, R. L.; Grönbeck, H.; Häkkinen, H. Proc. Natl. Acad. Sci. U.S.A. 2008, 105, 9157. 
levels (LUMO) ${ }^{4 \mathrm{~d}, \mathrm{j}, 8}$ These simple experiments also are a direct measure of MPC size dispersity in a given sample as each cluster has its own charging signature that is dependent on core size, nature of the protecting monolayer, and the dispersing medium. ${ }^{8,9}$ The kinetics of the electron transfer and the stability of the MPC upon electron removal or addition can also be readily addressed. ${ }^{8,10}$

There are numerous reports concerning the quantized charging of MPCs with a core atom count of ca. 140 atoms, $\mathrm{Au}_{\sim 140}{ }^{1,7,9 \mathrm{~b}, 10 \mathrm{a}, \mathrm{d}, 11}$ Its charging is well-understood, and it has been used as a platform to understand the fundamental properties of MPCs. ${ }^{1,3,9 a}$ With the exception of Au25, ${ }^{4 \mathrm{~d}, 8,12}$ MPCs with a core size smaller than $1 \mathrm{~nm}$ have proved to be more difficult to obtain in sufficient monodispersity for electrochemical experiments. Murray and co-workers reported the general synthesis and charging properties for Au25 with a variety of protecting monolayers and recently reported its crystal structure. ${ }^{4 \mathrm{a}, 8 \mathrm{~b}, 12 \mathrm{~b}, 13}$ High-yield synthesis methods have

(5) (a) Wang, L. Y.; Shi, X. J.; Kariuki, N. N.; Schadt, M.; Wang, G. R.; Rendeng, Q.; Choi, J.; Luo, J.; Lu, S.; Zhong, C. J. J. Am. Chem. Soc. 2007, 129, 2161. (b) Rowe, M. P.; Steinecker, W. H.; Zellers, E. T. Anal. Chem. 2007, 79, 1164. (c) Krasteva, N.; Fogel, Y.; Bauer, R. E.; Mullen, K.; Matsuzawa, N.; Yasuda, A.; Vossmeyer, T. Adv. Funct. Mater. 2007, 17, 881. (d) Choi, J. P.; Coble, M. M.; Branham, M. R.; DeSimone, J. M.; Murray, R. W. J. Phys. Chem. C 2007, 111, 3778. (e) Ibanez, F. J.; Zamborini, F. P. Langmuir 2006, 22, 9789. (f) Ibanez, F. J.; Gowrishetty, U.; Crain, M. M.; Walsh, K. M.; Zamborini, F. P. Anal. Chem. 2006, 78, 753. (g) Ruiz, V.; Nicholson, P. G.; Jollands, S.; Thomas, P. A.; Macpherson, J. V.; Unwin, P. R. J. Phys. Chem. B 2005, 109, 19335. (h) Leopold, M. C.; Donkers, R. L.; Georganopoulou, D.; Fisher, M.; Zamborini, F. P.; Murray, R. W. Faraday Discuss. 2004, 125, 63. (i) Markovich, G.; Collier, C. P.; Henrichs, S. E.; Remacle, F.; Levine, R. D.; Heath, J. R. Acc. Chem. Res. 1999, 32, 415. (j) Shenhar, R.; Rotello, V. M. Acc. Chem. Res. 2003, 36, 549.

(6) Chen, S. W.; Ingram, R. S.; Hostetler, M. J.; Pietron, J. J.; Murray, R. W.; Schaaff, T. G.; Khoury, J. T.; Alvarez, M. M.; Whetten, R. L. Science 1998, 280, 2098.

(7) (a) Miles, D. T.; Murray, R. W. Anal. Chem. 2003, 75, 1251. (b) Hicks, J. F.; Miles, D. T.; Murray, R. W. J. Am. Chem. Soc. 2002, 124, 13322.

(8) (a) Antonello, S.; Holm, A. H.; Instuli, E.; Maran, F. J. Am. Chem. Soc. 2007, 129, 9836. (b) Jimenez, V. L.; Georganopoulou, D. G.; White, R. J.; Harper, A. S.; Mills, A. J.; Lee, D.; Murray, R. W Langmuir 2004, 20, 6864.

(9) (a) Garcia-Morales, V.; Mafé, S. J. Phys. Chem. C 2007, 111, 7242. (b) Quinn, B. M.; Liljeroth, P.; Ruiz, V.; Laaksonen, T.; Kontturi, K. J. Am. Chem. Soc. 2003, 125, 6644. (c) Miles, D. T.; Leopold, M. C.; Hicks, J. F.; Murray, R. W. J. Electroanal. Chem. 2003, 554, 87.

(10) (a) Quinn, B. M.; Liljeroth, P.; Kontturi, K. J. Am. Chem. Soc. 2002 124, 12915. (b) Peterson, R. R.; Cliffel, D. E. Langmuir 2006, 22, 10307. (c) Georganopoulou, D. G.; Mirkin, M. V.; Murray, R. W. Nano Lett. 2004, 4, 1763. (d) Laaksonen, T.; Pelliniemi, O.; Quinn, B. M. J. Am. Chem. Soc. 2006, 128, 14341.

(11) (a) Wuelfing, W. P.; Green, S. J.; Pietron, J. J.; Cliffel, D. E.; Murray, R. W. J. Am. Chem. Soc. 2000, 122, 11465. (b) Pietron, J. J.; Hicks, J. F.; Murray, R. W. J. Am. Chem. Soc. 1999, 121, 5565.

(12) (a) Kim, J.; Lema, K.; Ukaigwe, M.; Lee, D. Langmuir 2007, 23, 7853. (b) Lee, D.; Donkers, R. L.; Wang, G.; Harper, A. S.; Murray, R. W. J. Am. Chem. Soc. 2004, 126, 6193.

(13) (a) Donkers, R. L.; Lee, D.; Murray, R. W. Langmuir 2008, 24, 5976 (b) Dass, A.; Stevenson, A.; Dubay, G. R.; Tracy, J. B.; Murray, R. W. J. Am. Chem. Soc. 2008, 130, 5940. (c) Dass, A.; Guo, R.; Tracy, J. B.; Balasubramanian, R.; Douglas, A. D.; Murray, R. W. Langmuir 2008, 24, 310. (d) Tracy, J. B.; Crowe, M. C.; Parker, J. F.; Hampe, O.; Fields-Zinna, C. A.; Dass, A.; Murray, R. W. J. Am. Chem. Soc. 2007, 129, 16209. (e) Wang, G.; Guo, R.; Kalyuzhny, G.; Choi, J. P.; Murray, R. W. J. Phys. Chem. B 2006, 110, 20282. (f) Choi, J. P.; Murray, R. W. J. Am. Chem. Soc. 2006, 128, 10496. (g) Wang, W.; Murray, R. W. Langmuir 2005, 21, 7015. (h) Song, Y.; Harper, A. S.; Murray, R. W. Langmuir 2005, 21, 5492. (i) Guo, R.; Song, Y.; Wang, G.; Murray, R. W. J. Am. Chem. Soc. 2005, 127, 2752. (j) Guo, R.; Murray, R. W. J. Am. Chem. Soc. 2005, 127, 12140. (k) Donkers, R. L.; Lee, D.; Murray, R. W. Langmuir 2004, 20, 1945. also been recently reported for Au25. ${ }^{4 \mathrm{~d}, 12 \mathrm{a}, 14}$ Discrete charging of Au9, Au11, Au13, Au38, Au55, and Au75 clusters has also been reported. ${ }^{6,9 \mathrm{~b}, 15}$ Pioneering work by Tsukuda and co-workers on MPC size separation using electrophoresis of glutathionate-protected clusters has revealed a broad range of core nuclearities between the limits of Au10 and Au39. ${ }^{4 b, c, f, g, i, 16}$ However their electrochemical quantized charging cannot be accessed as the MPCs are water-soluble. ${ }^{9 a}$

Here, we report a simple synthesis strategy to obtain hexanethiolate-protected Au38 based on its stability in excess thiol. The reduction time in the standard Brust-Schiffrin two-phase synthesis ${ }^{2}$ was optimized such that Au38 were the only clusters that were fully passivated by the thiol monolayer. As previously noted, decreasing the synthesis temperature and shortening the reduction times generates a higher proportion of MPCs with subnanometer core diameters. ${ }^{4 \mathrm{~d}, 12 \mathrm{a}, \mathrm{b}, 13 \mathrm{k}, 14}$ Under the conditions employed, larger particles formed were vulnerable to etching by excess thiol and were removed from solution. However, unlike previous reports, ${ }^{4 \mathrm{~d}, 12 \mathrm{a}, \mathrm{b}, 13 \mathrm{k}, 14}$ our synthesis yields Au38 and not Au25. The presence of Au25 was not apparent in any of the mass spectra or electrochemical data obtained pre- or post-treatment of the MPC solutions with excess thiol. The reason for this is speculative and may be due to the thermodynamic stability of Au38 versus the kinetic stability of Au25. ${ }^{4 \mathrm{~g}, 14,17}$ The isolated Au38 was characterized by mass spectrometry, optical spectroscopy, and electrochemical techniques. With the use of the latter, the stability of Au38 is unequivocally demonstrated to be dependent on the charge stored in the core.

This latter point is of particular interest as there is renewed impetus in understanding the gold-thiol chemistry underpinning the stability of MPCs following the recent total structure determination of an MPC by Jadzinsky et $a .^{4 \mathrm{e}}$ In their singlecrystal determination of $\mathrm{Au}_{102}(\mathrm{RS})_{44}$, it was revealed that the binding motif is more complex than the conventional selfassembled monolayer (SAM) picture based on simple $\mathrm{Au}-\mathrm{S}$ bonds. The MPC was revealed to have a $79 \mathrm{Au}$ atom core with oligomeric $\mathrm{Au}(\mathrm{I})$ - thiolate complexes as the outer shell. ${ }^{4 e, 41} \mathrm{~A}$ similar motif was also predicted ${ }^{18}$ and experimentally verified for $\mathrm{Au}_{25}(\mathrm{RS})_{18}{ }^{4 \mathrm{a}, 19}$ It is likely that this binding motif is generally applicable to all SAMs whether formed on nano or macro gold surfaces. The stability of SAMs formed on macroscopic surfaces has been widely investigated, and the desorption of the monolayer under reducing conditions is well-understood. ${ }^{3 a}$ This phenomenon is less well-understood for SAMs on nanoparticle surfaces. This is mostly due to the fact that the SAM is integral to the nanoparticle stability in solution and if even a fraction of the monolayer is removed, the nanoparticles will aggregate irreversibly. ${ }^{1}$ Desorption of thiolates from the reduced MPC has

(14) Zhu, M.; Lanni, E.; Garg, N.; Bier, M. E.; Jin, R. J. Am. Chem. Soc. 2008, 130, 1138 .

(15) (a) Wen, F.; Englert, U.; Gutrath, B.; Simon, U. Eur. J. Inorg. Chem. 2008, 106. (b) Menard, L. D.; Gao, S. P.; Xu, H. P.; Twesten, R. D.; Harper, A. S.; Song, Y.; Wang, G. L.; Douglas, A. D.; Yang, J. C.; Frenkel, A. I.; Nuzzo, R. G.; Murray, R. W. J. Phys. Chem. B 2006, 110, 12874. (c) Balasubramanian, R.; Guo, R.; Mills, A. J.; Murray, R. W. J. Am. Chem. Soc. 2005, 127, 8126. (d) Yang, Y.; Chen, S. Nano Lett. 2003, 3, 75.

(16) (a) Shichibu, Y.; Negishi, Y.; Tsukuda, T.; Teranishi, T. J. Am. Chem. Soc. 2005, 127, 13464. (b) Negishi, Y.; Takasugi, Y.; Sato, S.; Yao, H.; Kimura, K.; Tsukuda, T. J. Am. Chem. Soc. 2004, 126, 6518.

(17) Schaaff, T. G.; Whetten, R. L. J. Phys. Chem. B 1999, 103, 9394.

(18) Akola, J.; Walter, M.; Whetten, R. L.; Häkkinen, H.; Grönbeck, H. J. Am. Chem. Soc. 2008, 130, 3756.

(19) Zhu, M.; Aikens, C. M.; Hollander, F. J.; Schatz, G. C.; Jin, R. J. Am. Chem. Soc. 2008, 130, 5883. 
been reported for both Au147 and Au25. ${ }^{8 a, 20}$ In light of the recent breakthroughs in the structure determination, ${ }^{4 a, e, 19}$ the exact nature of the leaving group is unresolved as it is unlikely to be a simple thiolate as has been assumed in analogy with the electrochemical studies at planar SAM-modified Au electrodes. Here we use electrochemical measurements to assess the stability of Au38 as a function of the charge stored in the core. For core oxidation, electron transfer is reversible and the clusters are stable in the oxidized state. In contrast, core reduction is more complicated involving following chemical reactions as the monolayer is desorbed. The reduced clusters are not stable. Thus, in contrast to the recent reports for Au25, ${ }^{4 a, d}$ the preferential charge state for Au38 is not -1 .

\section{Experimental Section}

Chemicals. Hydrogen tetrachloroaurate trihydrate $\left(\mathrm{HAuCl}_{4}\right.$. $3 \mathrm{H}_{2} \mathrm{O}$, Sigma-Aldrich), tetraoctylammonium bromide (TOABr, Alfa Aesar), hexanethiol $\left(\mathrm{C}_{6} \mathrm{SH}\right.$, Alfa Aesar), sodium borohydride (Merck), toluene (Laboratory Scan), acetonitrile (ACN, Merck), 1,2dichloroethane (DCE, Riedel-de Haën), chloroform (Riedel-de Haën), and tetrachloroethane (TCE, Riedel-de Haën) were used as received. Bis(triphenylphosphoranylidene) ammonium tetrakis(pentafluorophenyl)borate $\left(\mathrm{BisTPPATPBF}_{20}\right.$ ), tetrabutylammonium hexafluorophosphate $\left(\mathrm{TBAPF}_{6}\right)$, and tetrabutylammonium perchlorate $\left(\mathrm{TBAClO}_{4}\right)$ were prepared as previously described. ${ }^{10 \mathrm{~d}}$ All other chemicals used were of the highest available commercial purity and were used as received.

Synthesis. Hexanethiolate MPCs were synthesized according to the Brust-Schiffrin two-phase method known to yield MPCs with small core diameters. ${ }^{2,4 k, 7,9 b, 21}$ Tetrachloroaurate was phasetransferred from aqueous solution to toluene using TOABr. A 3-fold molar excess of hexanethiol relative to gold was then added to the separated toluene phase. The solution was cooled on an ice bath before the fast addition of a freshly prepared aqueous solution of sodium borohydride (10-fold molar excess with respect to $\mathrm{Au}$ reactant) under high stirring. The duration of the reduction step was varied from $15 \mathrm{~min}$ to several days. Typically the reduction was allowed to proceed interrupted for a given period of time. In some instances, aliquots were taken from the synthesis solution at defined times. The phases were then separated, and the particles were precipitated from the toluene solution by the addition of excess ACN. The precipitate was recovered from solution and washed well with ACN. The as-prepared particles were then dispersed in DCE and exposed to excess hexanethiol under stirring for periods varying from 3 days to 2 weeks. Typically, several successive thiol treatments were required until the desired degree of monodispersity was achieved. Particles were cleaned by precipitation/dispersion cycles as previously described. ${ }^{10 \mathrm{~d}}$

MPC Characterization. Matrix-assisted laser desorption ionization time-of-flight (MALDI-TOF) mass spectrometry was used to determine the dispersity of core masses. The MPC solution ( 0.54 $\mu \mathrm{g} / \mu \mathrm{L}$ in toluene) was pipetted onto the sample plate together with the $\alpha$-cyano-4-hydroxycinnamic acid matrix and air-dried. The MALDI-TOF mass spectrum was obtained using an Ultraflex TOF/ TOF instrument (Bruker-Daltonik GmbH, Bremen, Germany) equipped with an $337 \mathrm{~nm}$ UV nitrogen laser. The instrument was externally calibrated using a standard mixture of proteins from Bruker. The MPC molecular formula was determined using thermogravimetric analysis (TGA) in conjunction with the mass spectrum. ${ }^{22}$ TGA was performed using a NETZSCH STA 449C

(20) Quinn, B. M.; Kontturi, K. J. Am. Chem. Soc. 2004, 126, 7168

(21) Schaaff, T. G.; Shafigullin, M. N.; Khoury, J. T.; Vezmar, I.; Whetten, R. L. J. Phys. Chem. B 2001, 105, 8785.

(22) Hostetler, M. J.; Wingate, J. E.; Zhong, C.-J.; Harris, J. E.; Vachet, R. W.; Clark, M. R.; Londono, J. D.; Green, S. J.; Stokes, J. J.; Wignall, G. D.; Glish, G. L.; Porter, M. D.; Evans, N. D.; Murray, R. W. Langmuir 1998, 14, 17. thermobalance. The dried sample was heated from 20 to $600{ }^{\circ} \mathrm{C}$ at a heating rate of $10{ }^{\circ} \mathrm{C} / \mathrm{min}$ under dynamic nitrogen flow of 40 $\mathrm{mL} / \mathrm{min}$. The UV-vis-NIR spectra of the dispersed Au38 MPCs were recorded using a Perkin-Elmer Lambda 950 UV-vis-NIR spectrometer. The particles were dispersed in TCE at a concentration of $0.11 \mu \mathrm{g} / \mu \mathrm{L}$.

Electrochemical Measurements. MPC core size and dispersity were first estimated using electrochemical measurements. Cyclic voltammetry (CV), differential pulse voltammetry (DPV), and square wave voltammetry (SWV) were performed in DCE-dispersed MPC solutions with $10 \mathrm{mM}$ BisTPPATPBF 20 as supporting electrolyte using a CHI 900 potentiostat (CH Instruments, Austin, TX). Oxygen was purged from solution by bubbling with $\mathrm{N}_{2}$, and a blanket of $\mathrm{N}_{2}$ was maintained over the solution for the duration of the measurements. In microelectrode measurements, a $25 \mu \mathrm{m}$ diameter Pt electrode was used as the working electrode (WE) and a Ag wire as both quasi-reference (QRE) and counter electrode. In macroelectrode measurements, a Pt wire was used as counter electrode, a Ag wire as QRE, and a $2 \mathrm{~mm}$ diameter Pt disk as the WE. Prior to the measurements, the WE was polished with 0.3 and $0.05 \mu \mathrm{m} \mathrm{Al} \mathrm{Al}_{2} \mathrm{O}_{3}$ slurries, rinsed well with water, and then sonicated in both ethanol and water. A background $\mathrm{CV}$ was always recorded to ensure that the $\mathrm{CV}$ was featureless through the entire potential window. $\mathrm{TBAClO}_{4}$ and $\mathrm{TBAPF}_{6}$ were also used as the base electrolytes, and the electrochemical responses from the particles were identical to that obtained with BisTPPATPBF 20 as base electrolyte.

Scanning electrochemical microscopy (SECM) experiments were performed with a commercial instrument $(\mathrm{CH}$ Instruments, Austin, TX). All measurements were performed in a $\mathrm{N}_{2}$-filled glovebag. A DCE-dispersed MPC solution with $10 \mathrm{mM}$ BisTPPATPBF $_{20}$ as supporting electrolyte was purged of $\mathrm{O}_{2}$ prior to being taken in the bag. Pt microelectrodes, 10 and $25 \mu \mathrm{m}$ diameter, with a known RG (ratio of the radii of the insulating sheath and the electrode) were used as the SECM tips. They were characterized by fitting experimental approach curves recorded to conducting and insulating substrates using ferrocene methanol as the redox mediator to theoretical plots for pure positive and negative feedback, respectively. ${ }^{23} \mathrm{~A} \mathrm{Ag} / \mathrm{AgCl}$ wire served as both counter electrode and QRE. SECM approach curves at an approach rate of $1 \mu \mathrm{m} \mathrm{s}^{-1}$ were obtained by biasing the tip at the diffusion-limited potential for the oxidation/reduction of Au38 and recording the tip current as a function of distance to the substrate. The potentials applied were determined based on CVs recorded at the tip. The substrate electrode was unbiased, and its potential is fixed through the Nernst equation at the equilibrium potential of the redox mediator used, here either Au38 or ferrocene methanol. ${ }^{10 a}$

Bulk electrolysis was undertaken in a three-compartment cell as previously described. ${ }^{10 \mathrm{a}, 11 \mathrm{~b}}$ The electrolysis was performed in a $\mathrm{N}_{2}$-filled glovebag, and solutions were purged of $\mathrm{O}_{2}$ using $\mathrm{N}_{2}$ prior to being taken into the bag. Au38 was dispersed in a DCE solution with $100 \mathrm{mM} \mathrm{TBAPF}_{6}$ as the base electrolyte. Microelectrode voltammetry and equilibrium potential, $E_{\text {eq }}$, of the particles at a $\mathrm{Pt}$ electrode versus the QRE were used to follow the extent of the electrolysis. The shift in $E_{\text {eq }}$ relative to the oxidation and reduction waves (peaks in the $\mathrm{d} I / \mathrm{d} E$ response) was used as a measure of the change in the charge of the particles in the bulk solution. Twophase oxidation and reduction of DCE-dispersed particles were carried out as previously described ${ }^{4 \mathrm{~d}, 11 \mathrm{a}}$ An acidic solution of $\mathrm{Ce}(\mathrm{IV})$ was used as the aqueous oxidant, and freshly prepared solutions of $\mathrm{NaBH}_{4}$ were used as the aqueous reductant. ${ }^{4 d}$ The concentration of $\mathrm{NaBH}_{4}$ was varied over several orders of magni-

(23) (a) Bard, A. J.; Faulkner, L. R. Electrochemical Methods, Fundamentals and Applications, 2nd ed.; John Wiley \& Sons Inc.: New York, 2001. (b) Amphlett, J. L.; Denuault, G. J. Phys. Chem. B 1998, 102, 9946. 

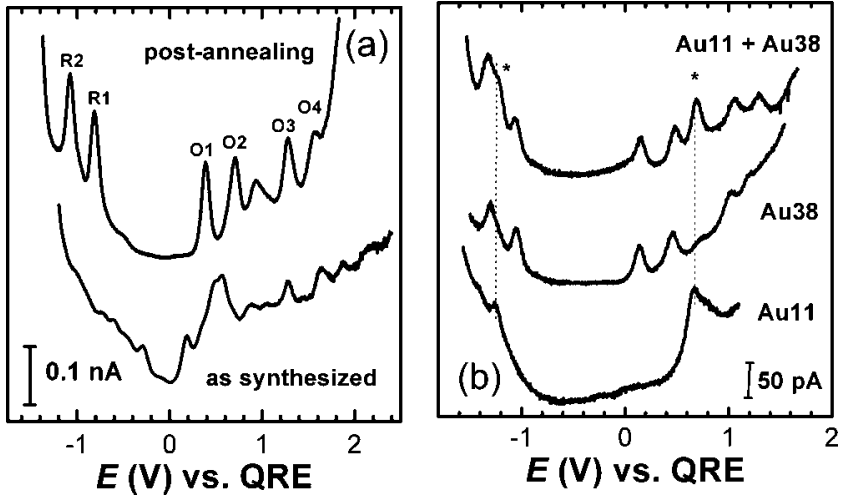

Figure 1. (a) DPVs of the MPCs from the shortest reduction time (ca. 15 min) compared with those post-annealed in excess thiol. (b) DPVs of DCEdispersed Au11, Au38, and a mixture of both cluster sizes. All DPVs were recorded at a $25 \mu \mathrm{m}$ diameter $\mathrm{Pt}$ microelectrode.

tude. The charge of the particles in the bulk solution was estimated as described above for the bulk electrolysis measurements.

\section{Results and Discussion}

As the resolution of single-electron charging peaks in electrochemical measurements such as DPV is a good indication of MPC monodispersity, ${ }^{7}$ this was used to see the influence of both the synthesis reduction time and the effect of the postsynthesis exposure to thiol. In Figure 1a, a microelectrode DPV of DCE-dispersed particles prepared using a very short reduction time is shown. The sharp peaks in the DPV at potential intervals typical for "molecular" MPCs ${ }^{9 b}$ are evidence of a dominant population of small core diameter particles. As the reduction time is increased, more peaks appeared in the DPV and the peak spacing between successive electron transfers, $\Delta E$, became more regular and characteristic of Au147. ${ }^{7,9 b}$ Variation of the reduction time used in the syntheses yielded a gradual evolution in core size. Typically, particles from the shortest reduction times had high populations of molecular MPCs as visualized by sharp peaks in the DPV response. At longer reduction times, the particles had a dominant population of Au147. This was representative for a synthesis allowed to proceed uninterrupted for a given reduction time. For aliquots taken from a single synthesis at different reduction times, a significant population of Au38 was also apparent in the long-time reduction particles ( $>6$ days). This may be due to the interruption of the stirring while taking samples at short times. Examples of SWV data obtained for particles synthesized at differing reduction times are given in the Supporting Information.

The monodispersity can be dramatically improved by subjecting the MPCs prepared using short reduction times ( $<30 \mathrm{~min}$ ) to an annealing step with excess thiol for long periods of time ( $>10$ days). This can be clearly seen in the upper DPV given in Figure 1a where two oxidation $(\mathrm{O} 1-\mathrm{O} 4)$ and one reduction (R1-R2) doublets are now clearly visible with a large voltage spacing between the first electron injection (R1) and removal (O1). This $1.2 \mathrm{~V}$ gap corresponds to a HOMO-LUMO gap of $0.9 \mathrm{~V}$ after correction for the charging energy estimated from the $\Delta E$ between the oxidation peaks $(\mathrm{O} 2-\mathrm{O} 1)$ as previously reported. ${ }^{9 \mathrm{~b}}$ This gap has been previously ascribed to a Au38 core. ${ }^{6,9 b, 13 a}$ As there are no peaks apparent within the $\mathrm{O} 1-\mathrm{R} 1$ gap, larger core diameters such as the ubiquitous Au147 are absent, indicating that exposure to excess thiol has effectively removed these MPCs from solution. There are, however, additional peaks apparent outside the central gap not charac-
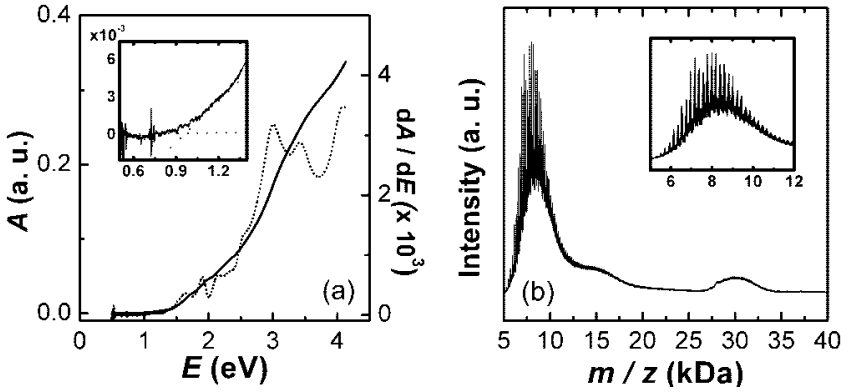

Figure 2. (a) Absorption spectrum (-) and the corresponding derivative $(\cdot \cdot \cdot)$ for $\mathrm{Au}_{38}\left(\mathrm{SC}_{6}\right)_{22}$ dispersed in tetrachloroethane (TCE). Inset: magnification of the zero-absorbance region. (b) MALDI-TOF mass spectrum of the post-annealed MPCs. The inset is a magnification of the group of peaks centered at $8.5 \mathrm{kDa}$.

teristic of Au38 indicating that smaller clusters are present. As can be seen in Figure 1b, there is a peak between the two Au38 oxidation doublets and a corresponding shoulder affecting the second Au38 reduction peak (denoted with an asterisk). A small quantity of these small clusters was isolated by size selection as Au38 tends to precipitate out of a DCE solution with time leaving the smaller particles in solution. Their electrochemical response is given in Figure 1b. The $1.9 \mathrm{~V}$ electrochemical gap is in the range previously reported for Au11-13 clusters $^{15 \mathrm{~b}, \mathrm{~d}}$ and is here denoted as Au11 as a first approximation. Attempts to isolate the particles from solution for further characterization were unsuccessful as the particles aggregated when dry. Kim et al. recently reported comparable electrochemical data and instability for small particles obtained during their optimization of the synthesis for Au25. ${ }^{12 a}$ The influence of these smaller particles on the response of Au38 is illustrated in Figure 1b where the responses for the monodisperse clusters are compared with that for a mixture.

On the basis of the electrochemical measurements, the relative populations of Au38 and Au11 do not change significantly with annealing. Thus, these clusters are remarkably resistant to etching with excess thiol and can be in solution with a hyperexcess of thiol (1000-fold) for weeks, and this can be repeated multiple times without any apparent change in the core size. Thus, smaller clusters, in particular Au38, are very stable against etching. Comparable stability against etching has been previously reported for Au25 $5^{4 \mathrm{c}}$ though here no Au25 clusters are apparent in either the electrochemical or mass spectral analysis of the post-annealed samples.

The number of thiols per particle was estimated using TGA. ${ }^{22}$ The fraction of organic material was 25.2\% (Supporting Information). Assuming a core composition of $\mathrm{Au} 38$, the corresponding molecular formula of the clusters is $\mathrm{Au}_{38}\left(\mathrm{SC}_{6}\right)_{22}{ }^{22}$ The absorption spectrum given in Figure 2a has an optical gap of $0.9 \mathrm{eV}$ estimated from the absorption onset consistent with the electrochemical gap and is identical to that previously reported for the $8 \mathrm{kDa}$ clusters. ${ }^{4 \mathrm{~b}, \mathrm{c}, \mathrm{k}}$ Taking the derivative of the spectra, peaks are apparent at 1.15, 1.62, 1.91, 2.15, 2.32, $2.58,3.01$, and $3.43 \mathrm{eV}$. These correlate well with those tabulated for the derivative spectra for $8 \mathrm{kDa}$ clusters by Schaaff et al. ${ }^{4 \mathrm{k}}$ The dispersity in core size pre- and post-annealing of the short synthesis time particles was accessed with MALDITOF mass spectrometry. ${ }^{24}$ All spectra obtained had a group of peaks centered at ca. $8.5 \mathrm{kDa}$ corresponding to $\mathrm{Au}_{38} \mathrm{~S}_{22}{ }^{-}$, whereas the number of other peak groups detected and their

(24) Schaaff, T. G. Anal. Chem. 2004, 76, 6187. 

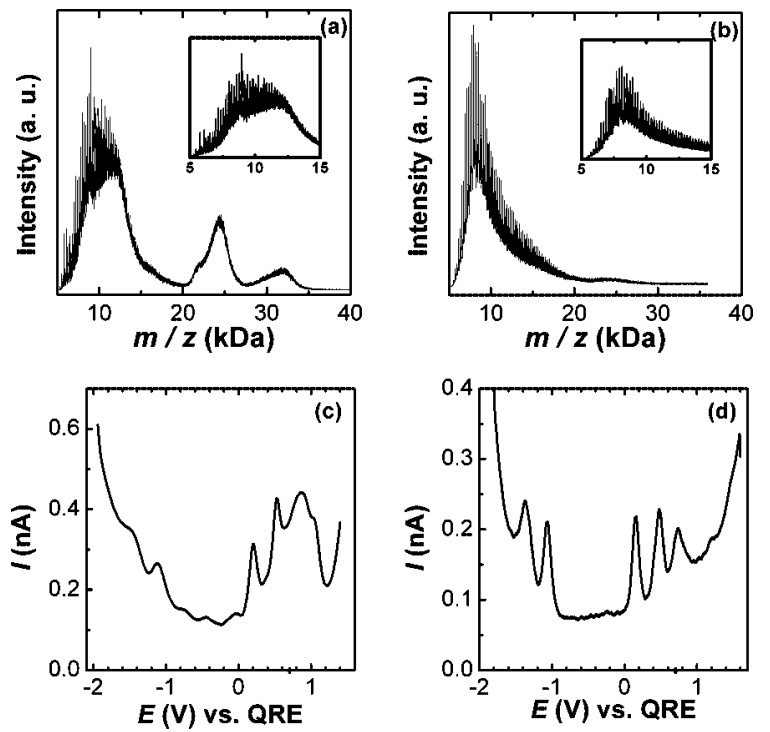

Figure 3. MALDI-TOF mass spectra of toluene solutions of the same particles pre- (a) and post- (b) annealing with excess thiol. The inset in both figures is a magnification of the low mass range. The corresponding SWVs recorded at a $25 \mu \mathrm{m}$ diameter Pt microelectrode for the pre- and post-annealed particles dispersed in DCE are given in (c) and (d), respectively.

relative intensities varied post-annealing. An example of a typical spectra obtained post-annealing is given in Figure $2 b$.

Figure 3 shows the correlation between electrochemical and MALDI-TOF mass spectrometry estimates of MPC polydispersity. In the electrochemical measurements, polydispersity is visualized as additional peaks in SWVs (Figure 3c) that disappear after the annealing process (Figure 3d). In the mass spectra, larger clusters are detected in the 22 and $29 \mathrm{kDa}$ regions (Figure 3a), whereas after annealing, only the group of peaks at $8.5 \mathrm{kDa}$ is observed (Figure $3 \mathrm{~b}$ ). The disappearance of the ca. 22 and $28 \mathrm{kDa}$ peak groups indicates that these larger cores disappear from solution with time in the presence of excess thiol (Figure 3b). Thus, the overlapping peaks apparent in the DPV for the as-synthesized particles shown in Figure 1a are due to the presence of ca. 8,22 , and $28 \mathrm{kDa}$ particles in solution. The abundance of low molecular weight fragments due to the MALDI conditions prevents detection of Au cores of mass less than $5 \mathrm{kDa}$.

As can be seen in Figure 3, exposing the particles obtained from the short-time syntheses to excess thiol has a marked effect on the dispersity in core size. Particles obtained from longer syntheses times did not show this effect upon annealing as illustrated in Figure 4a, where the SWV responses for particles from a 7 day preparation pre- and post-annealing are given. The pre-annealing sample was from a single synthesis where aliquots were taken at defined reduction times. This indicates that the larger particles from the long-time syntheses are stable in excess thiol in contrast to those obtained from the short-time syntheses. To verify that Au147 is stable to etching, a monodisperse preparation of Au147 (from an uninterrupted long-time synthesis) was also subjected to the same annealing as the Au38 particles and there was little difference in the electrochemical response for the particles pre- and postexposure to thiol (Figure 4b).

The disappearance of the larger MPCs from solution upon annealing the short-time synthesis particles is intriguing, and it is likely that the larger particles formed during the short synthesis times are not fully coated with thiol and are thus
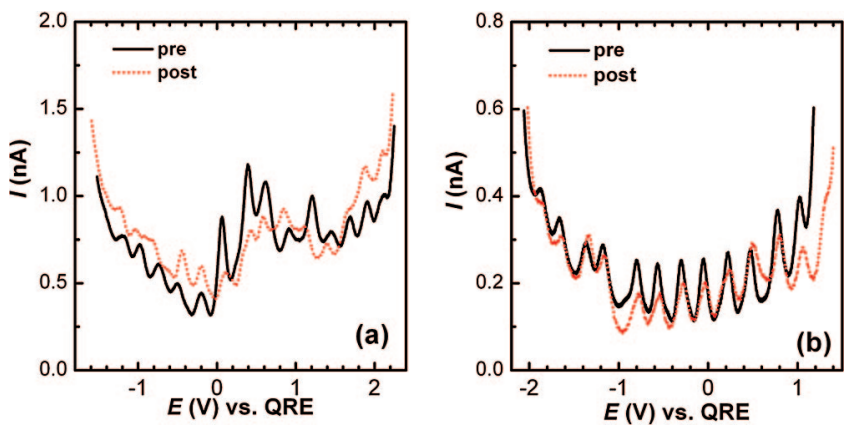

Figure 4. (a) Comparison of the SWV responses for dispersed particles at a Pt microelectrode obtained from long synthesis times pre- and postannealing with excess thiol. (b) Comparison of the SWV responses for monodisperse Au147 pre- and post-annealing with excess thiol.
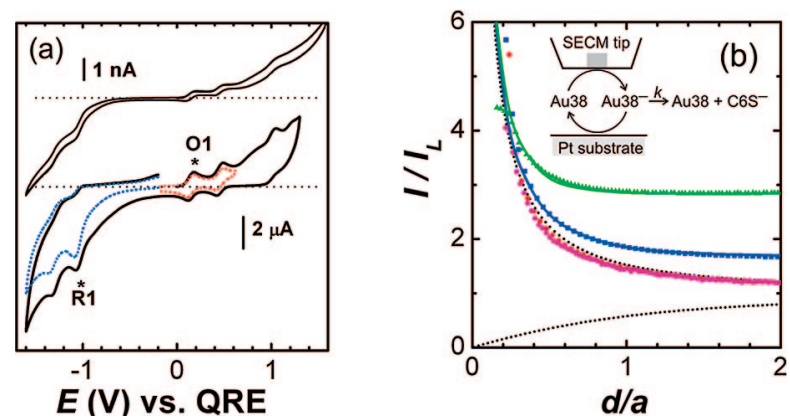

Figure 5. (a) CVs for DCE-dispersed Au38 at a Pt microelectrode (upper trace) and macroelectrode (lower trace). The dotted red (blue) lines are the single-scan oxidation (reduction) CVs from $E_{\text {eq. }}$. Peaks $\mathrm{O} 1$ and R1 are marked with an asterisk. Scan rate: $100 \mathrm{mV} \mathrm{s}^{-1}$. (b) SECM approach curves to a Pt substrate using $\mathrm{Au} 38^{z}$ as the redox mediator showing pure positive feedback for $z=+1$ for tip radii of 5 (red dots) and $12.5 \mu \mathrm{m}$ (magenta dots). For $z=-1$, the experimental (symbols) and corresponding fits (lines) to theory are shown for tip radii of 5 (blue squares) and $12.5 \mu \mathrm{m}$ (green triangles). The dotted lines show the theoretical curves for pure positive and pure negative feedback. For $z=-1, I / I_{\mathrm{L}}>1$ for all values of $d / a$ due to the EC mechanism. The inset is a schematic of the SECM operating principle.

susceptible to etching in the excess thiol solution. The smaller Au38 clusters are fully protected already at short synthesis times and cannot be etched by the excess thiol. A similar conclusion was reached based on the stability of Au25 to etching relative to its larger counterparts. ${ }^{4 c}$ Au38 does, however, tend to aggregate if stored in solution or dry with base electrolyte salt.

The influence of charge on the stability of the cluster is very relevant to their underlying structure. ${ }^{4 \mathrm{~d}}$ Here, we demonstrate that electrochemical measurements offer a particularly sensitive and direct means of probing this. On the basis of voltammetry on both micro- and macroelectrodes, it is apparent that the reduced particles are not as stable as their oxidized counterparts. Microelectrode CVs show that reduction currents for R1 and $\mathrm{R} 2$ are typically greater than those for the corresponding $\mathrm{O} 1$ and $\mathrm{O} 2$ oxidations (Figure 5a upper trace), which is not the case for the larger Au147. ${ }^{7,9 b} \mathrm{CV}$ s recorded at a macroelectrode (Figure 5b lower traces) also indicate that although Au38 oxidation is a simple one-electron diffusion-limited reaction giving Nernstian peaks in the forward and reverse scans, the reduction is more complicated. The reverse scan peaks are completely absent for both $\mathrm{R} 1$ and $\mathrm{R} 2$ at low scan rates indicating that the reduced species is no longer available in solution to be reoxidized at the electrode surface. The peak current for R1 is also significantly greater than that for O1. The 
scan rate dependence for macroelectrode $\mathrm{CVs}$ is given in the Supporting Information.

The CVs are analogous to those reported recently for Au25 where it was revealed that the initial core reduction triggers a cascade of chemical and electrochemical reactions where a thiolate molecule $\left(\mathrm{C}_{6} \mathrm{~S}^{-}\right)$dissociates from the reduced core and the resulting neutral metal core can accept another electron at the same potential. ${ }^{8 \mathrm{a}}$ Recent Au25 crystal structure determination revealed that the core is in fact composed of $13 \mathrm{Au}$ atoms protected by $6(\mathrm{RS})_{3} \mathrm{Au}_{2}$ oligomers. ${ }^{4 a, 19}$ Thus the leaving group in the following chemical reaction is unlikely to be a simple thiolate. Au38 is likely to have a similar "divide and protect" bonding motif. ${ }^{41,18}$ However, as the exact nature of the desorbing species has not been resolved, we use the simple thiolate description here as a first approximation. The dissociative electron transfer results in a cascade reaction $(\mathrm{EC})_{n}$ where $n$ is the number of thiolates desorbed:

$$
\begin{gathered}
\mathrm{Au}_{38}\left(\mathrm{SC}_{6}\right)_{22}+\mathrm{e}^{-} \rightarrow \mathrm{Au}_{38}\left(\mathrm{SC}_{6}\right)_{22}{ }^{-} \\
\mathrm{Au}_{38}\left(\mathrm{SC}_{6}\right)_{22}{ }^{-} \rightarrow \mathrm{Au}_{38}\left(\mathrm{SC}_{6}\right)_{21}+\mathrm{C}_{6} \mathrm{~S}^{-} \\
\mathrm{Au}_{38}\left(\mathrm{SC}_{6}\right)_{21}+\mathrm{e}^{-} \rightarrow \mathrm{Au}_{38}\left(\mathrm{SC}_{6}\right)_{21}{ }^{-}
\end{gathered}
$$

and so on where we assume that every chemical reaction step has a rate constant of $k$ and that all the electrochemical steps are fast and have the same standard potential. The shape of macroelectrode CVs is very sensitive to $k, n$, and the sweep rate $v$ as shown by simulated CVs in the Supporting Information. Moderate $k$ and $n$ reproduce the shape of the experimental CVs, but it is difficult to fully reproduce their sweep rate dependence. Consequently, it is very difficult to independently estimate $k$ and $n$ from the CVs. On the basis of the observed response, it is clear that $n$ is larger than 2 or 3 ; at the same time, the stability of the clusters in solution requires that $n$ cannot be larger than $\sim 10$. Desorption of a significant fraction of the thiolates would also notably change the standard potential of the electrochemical steps in contrast to our assumption. In addition, the shoulder apparent before peak (wave) R1 in the macro (micro) electrode CVs made it difficult to determine the peak (diffusion-limited) current unambiguously. The origin is speculative as it scaled with the Au38 concentration and is thus not due directly to residual $\mathrm{O}_{2}$ in solution or a contaminant in the solvent/base electrolyte system. Also, it should be noted that although the current ratio of $\mathrm{R} 1$ to $\mathrm{O} 1$ was always greater than 1 , there were deviations between measurements. This is likely to be caused by the presence of free thiol or thiolate that can affect the chemical steps through the law of mass action.

In order to obtain more information on the mechanism and the rate constant of the homogeneous chemical reaction, we use the particles as the redox mediator in SECM experiments (inset of Figure 5b). ${ }^{10 a-c}$ Au $38^{z}$ was generated at the SECM tip, and approach curves were measured by monitoring the diffusionlimited tip current (normalized by the limiting current far from the substrate in the absence of homogeneous chemical reactions, $I / I_{\mathrm{L}}$ ) as a function of the distance, $d$, between the tip and the substrate. ${ }^{10 a}$ As expected, approach curves to a Pt substrate for $z=+1$ and +2 gave pure positive feedback for tip radii, $a$, of 12.5 and $5 \mu \mathrm{m}$ and fitted well to theoretical plots for diffusionlimited positive feedback (Figure 5b). In contrast, identical measurements for $z=-1$ and -2 do not give pure positive feedback and the extent of deviation was dependent on the tip size with the larger tip showing less positive feedback (Figure $5 b)$. This difference has to do with enhancement of mass transfer as the electrode size is reduced; this reduces the effect of homogeneous chemical reactions on the SECM response. ${ }^{23 a} \mathrm{We}$ have modeled the SECM response according to the mechanism given above for small $n(2,3$, or 4$)$ and at the limit of large $n$. The SECM approach curves clearly fit the large $n$ limit better than $n=2,3$, or 4 (details are given in the Supporting Information). The approach curves for both SECM tip sizes can be fitted with the same rate constant of the homogeneous chemical step (Figure 5b), which gives $k=8 \mathrm{~s}^{-1}$.

In summary, voltammetry at both micro and macroelectrodes and SECM approach curves are all consistent with a simple one-electron reversible mechanism for $\mathrm{O} 1$ and a more complicated $(\mathrm{EC})_{n}$ mechanism for R1 with rate constant for the homogeneous step of $k=8 \mathrm{~s}^{-1}$. This rate is smaller than that estimated for Au25; ${ }^{8 a}$ although this can be rationalized by the higher curvature of the Au25 surface compared to Au38, the nature of the leaving group for both clusters needs to elucidated for a full understanding of this difference. ${ }^{4 a, e, 1}$

To probe the long-term stability of the charged species, bulk electrolysis ${ }^{11 \mathrm{~b}}$ and two-phase oxidation-reduction ${ }^{4 \mathrm{~d}}$ were used. Microelectrode voltammetry and the position of the equilibrium potential, $E_{\text {eq }}$, relative to the oxidation and reduction waves were used to determine the outcome of the processes. ${ }^{11 \mathrm{~b}}$ The as-prepared particles and their annealed counterparts have a charge of zero based on $E_{\text {eq, }}$ being within the HOMO-LUMO gap. Microelectrode voltammetry also clearly shows that the species in solution is $\mathrm{Au} 38^{\circ}$ as the zero-current line is between the $-1 / 0$ and $0 /+1$ waves. Au $38^{0}$ can be readily converted to $\mathrm{Au} 38^{+}$and $\mathrm{Au} 38^{2+}$ by both bulk electrolysis or two-phase oxidation. The shift in $E_{\text {eq }}$ past the oxidation waves and the position of the zero line in microelectrode CV (Supporting Information) confirms the success of the electrolysis. In contrast, two-phase reduction of $\mathrm{Au} 38^{\circ}$ using $\mathrm{NaBH}_{4}$ did not change the charge state of the bulk particles from 0 to -1 despite using vast excesses of the reductant. $E_{\text {eq }}$ remained within the gap, and the particles were not reduced. Bulk electrolysis of either oxidized or neutral particles to generate $\mathrm{Au} 38^{-}$was not successful as the reduced particles were not detected in solution postelectrolysis. The microelectrode CV lost the characteristic features of Au38 as the electrolysis proceeded. The duration of the bulk electrolysis experiment should be sufficient to strip off the protecting thiolate monolayer leaving the core vulnerable to aggregation. Then, the removal of even a third of the thiolates would have a profound influence on the MPC capacitance and thus the peak spacing between the $\mathrm{O} 1-\mathrm{O} 2$ and $\mathrm{R} 1-\mathrm{R} 2$ peaks.

There is an important caveat to using the $E_{\text {eq }}$ to determine the charge of the particles in solution as it is based on the assumption that the first reduction and first oxidation correspond to $-1 / 0$ and $0 /+1$ charge states, respectively. If the cluster has an odd number of electrons (e.g., Au25), then there will be a singly occupied molecular orbital (SOMO). In that case, for a neutral cluster, this would imply that the first reduction peak corresponds to the addition of an electron to the SOMO, whereas oxidation corresponds to removing the electron from the same level. The peaks should then be separated by the charging energy only. However, this is not consistent with the experiment: CVs of Au25 show a large gap between the first oxidation and reduction peaks. ${ }^{8 \mathrm{a}, \mathrm{b}, 13 \mathrm{a}, \mathrm{k}}$ The electrochemical experiments can be understood if Au25 is initially charged (e.g., -1) as has been recently verified using ESI mass spectrometry, ${ }^{4 \mathrm{~d}, 13 \mathrm{~d}} \mathrm{X}$-ray crystal structure, ${ }^{4 \mathrm{a}}$ and density functional theory (DFT) calculations. ${ }^{18,19}$ This implies that the reductive desorption of the thiolate species from Au25 and Au38 is initiated at charge states -2 and -1 , 
respectively. It should be reiterated that this phenomenon is not unexpected as it is analogous to reductive desorption of thiol SAMs from planar Au electrodes ${ }^{3 a}$ and has already been reported for other Au MPCs. ${ }^{8 a, 20}$ The nature of the leaving species still remains to be elucidated as it is now generally accepted that the thiolates are in fact $\mathrm{Au}$-thiolate complexes. ${ }^{4 a, e, 1,18,19}$

\section{Conclusions}

We have developed a facile synthesis strategy to obtain monodisperse hexanethiolate-protected $\mathrm{Au}_{38}\left(\mathrm{SC}_{6}\right)_{22}$ clusters based on resistance to etching upon exposure to a hyperexcess of thiol. We demonstrated that electrochemical experiments are uniquely suited to probe the electronic structure of the clusters and provide a direct measure of the cluster stability at a given charge. We report that Au38 can be reversibly oxidized to $z=$ +1 or +2 . However, in contrast to Au25 clusters, reduction of Au38 to $z=-1$ leads to desorption of the protecting thiolate monolayer. Recent experiments and theoretical calculations have revealed that the binding motif of the thiolate monolayer on the cluster surface is more complex than the conventional SAM picture based on simple $\mathrm{Au}-\mathrm{S}$ bonds. Molecular-level understanding of the difference in cluster stability and the mechanism of thiolate desorption has to incorporate the structural details of the complex $\mathrm{Au}-\mathrm{S}$ interface.

Acknowledgment. This research was funded by the Academy of Finland and NWO (P.L., Vidi-Grant 700.56.423). We thank H. Häkkinen for useful discussions and J. Malm for performing the TG measurement.

Supporting Information Available: Additional macroelectrode CVs, SWV for particles from different synthesis reduction times, examples of the correlation between mass spectrometry and electrochemical estimations of particle dispersity, and details of modeling of CVs and SECM approach curves. This material is available free of charge via the Internet at http://pubs.acs.org.

JA802317T 